\title{
Klassifikation und Benennung von Myopathien
}

Stephan Neudecker,

Klinik und Poliklinik für Neurologie, Martin-Luther-Universität Halle-Wittenberg, Halle/Saale

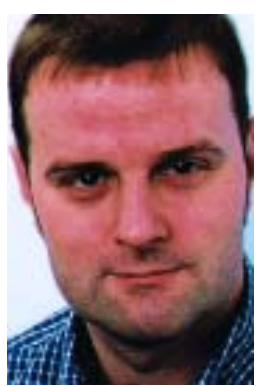

Stephan Neudecker

psychoneuro 2003; 29 (3): 86-93

\section{Abb. 1 Schematische Darstellung typischer dystrophischer Skelettmuskelveränderungen}

Destohr/fritsdurif ENervaheillounde Bil.1.

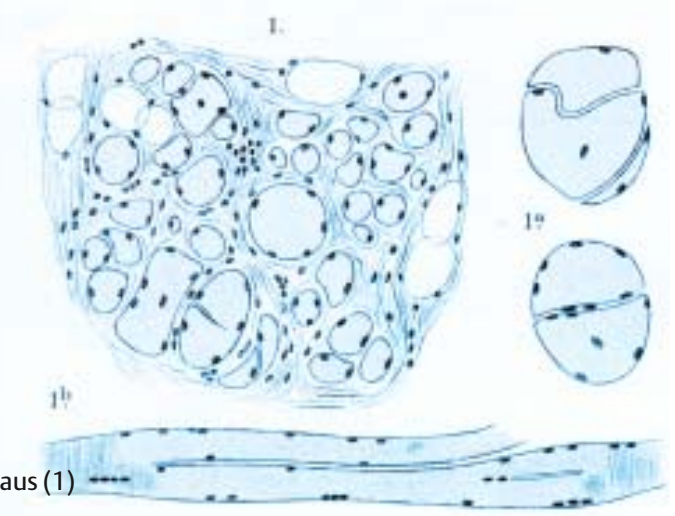

A b der ersten Hälfte des 19. Jahrhunderts mehrten sich Berichte über Patienten mit im Vordergrund der Symptomatik stehenden Atrophien und Paresen der Skelettmuskulatur. Die Genese eines Muskelschwunds wurde zunächst in Veränderungen der motorischen Vorderhornzellen vermutet und die Erkrankung(en) als „progressive Muskelatrophie(n)“ bezeichnet.

In den folgenden Jahrzehnten wurde immer offensichtlicher, dass von neurogenen Muskelatrophien Erkrankungen abzugrenzen sind, die auf einer primär myopathischen Ursache beruhen. In der zweiten Hälfte des 19. Jahrhunderts konnten verschiedene Myopathien beschrieben werden, die zunächst nach ihrem Erstbeschreiber (z.B. Duchenne-Muskeldystrophie), nach dem jeweiligen Phänotyp (z.B. fazioskapulohumerale Muskeldystrophie), anhand des Erkrankungsbeginns (z.B. späte juvenile Muskel- dystrophie) und/oder einer Kombination aus mehreren der genannten Kriterien (z.B. juvenile skapulohumerale Muskeldystrophie Erb) benannt wurden. Der Begriff „progressive Muskeldystrophie“ wurde ab 1884 von Wilhelm Erb eingeführt (1). Erb wollte damit bei dieser Erkrankungsgruppe - im Unterschied zu den Muskelatrophien - auf das klinische und histopathologische Nebeneinander von Hypertrophie und Atrophie sowie auf weitere myopathologische Veränderungen wie Kernvermehrung, Spaltbildung und Vakatwucherung des endomysialen Binde- und Fettgewebes hinweisen (Abb. 1), ohne damit eine sichere primär myogene Ursache postulieren zu wollen.

Die erste allgemein akzeptierte und für lange Zeit gültige Klassifikation von Myopathien lieferten Mitte der 50er-Jahre Walton und Natrass (8). Diese stellten den bis dahin bekannten Muskeldystrophien auf der- 
selben Hierarchieebene u.a. verschiedene, ebenfalls hereditäre und durch besondere klinische Phänomene charakterisierte Myotonien (z.B. Dystrophia myotonica) sowie einige erworbene Muskelkrankheiten (z.B. thyreotoxische Myopathie) gegenüber.

Fortschritte in der biochemischen Diagnostik ermöglichten ab der zweiten Hälfte des 20. Jahrhunderts die Identifizierung verschiedener Myopathien, die auf Defekte von Glykogen-, Glukose- und Lipidstoffwechsel beruhen und als metabolische Myopathien bezeichnet wurden. Die jeweiligen Krankheitsentitäten wurden - teilweise auch synonym - nach dem zugrunde liegenden Enzymdefekt (z.B. Myophosphorylase-Mangel), nach dem Erstbeschreiber (z.B. McArdle-Erkrankung) oder numerisch in der Reihenfolge ihrer Entdeckung (z.B. Glykogenose Typ V) benannt.

Neue Möglichkeiten der myohistologischen Aufarbeitung und Untersuchung führten ebenso seit den 1950er-Jahren zu Beschreibungen von früh beginnenden Muskelerkrankungen mit vergleichbarem Phänotyp und jeweils charakteristischen, namensgebenden Strukturveränderungen (z.B. Central coreMyopathie, Abb. 2), die in der Folge als kongenitale Myopathien zusammengefasst und als Krankheitsgruppe von den Muskeldystrophien abgegrenzt wurden.

Hinsichtlich erworbener Muskelerkrankungen gelang der Nachweis, dass entzündliche Veränderungen in der Muskulatur Ausdruck unterschiedlicher Erkrankungen und Pathomechanismen sein können, die zur Gruppe der Myositiden oder Muskelentzündungen zusammmengefasst wurden.

Seit Anfang der 80er-Jahre des 20. Jahrhunderts konnten verschiedene Erkrankungen auf eine mitochondriale Genese zurückgeführt werden. Klinisch können sich derartige Mitochondriopathien u.a. als Myopathien und Enzephalomyopathien wie auch in Form von Multisystemerkrankungen präsentieren. Im Jahre 1988 gelang der Nachweis pathogener Veränderungen des mitochondrialen Genoms und eröffnete neue Möglichkeiten der Diagnostik (3). Mitochondriale Erkrankungen erhielten ihre Namen entweder nach der im Vordergrund stehenden Sympexterne Ophthalmoplegie), nach dem Erstbeschreiber (z.B. Leigh-Syndrom) oder werden mit einem Akronym be-

\section{Tab. 1 Hereditäre Myopathien -Teil I: Muskeldystrophien}

\begin{tabular}{|c|c|c|}
\hline Untergruppe & Krankheit & Gendefekt \\
\hline \multirow[t]{2}{*}{ Dystrophinopathien } & Muskeldystrophie Duchenne & Dystrophin \\
\hline & Muskeldystrophie Becker & Dystrophin \\
\hline \multirow[t]{3}{*}{ Kernhüllenmyopathien } & Muskeldystrophie Emery-Dreifuss & Emerin \\
\hline & Muskeldystrophie & \\
\hline & Hauptmann-Thannhauser & Lamin $\mathrm{A} / \mathrm{C}$ \\
\hline \multirow{18}{*}{$\begin{array}{l}\text { Gliedergürteldystrophien } \\
\text { (LGMD) }\end{array}$} & LGMD1A (Myotilinopathie) & Myotilin \\
\hline & LGMD1B (Laminopathie) & Lamin A/C \\
\hline & LGMD1C (Caveolinopathie) & Caveolin 3 \\
\hline & LGMD1D & $?$ \\
\hline & LGMD1E & (Filamin 2?) \\
\hline & LGMD2A (Calpainopathie) & Calpain 3 \\
\hline & LGMD2B (Dysferlinopathie) & Dysferlin \\
\hline & LGMD2C ( $\gamma$-Sarkoglykanopathie) & $\gamma$-Sarkoglykan \\
\hline & LGMD2D ( $\alpha$-Sarkoglykanopathie) & $\alpha$-Sarkoglykan \\
\hline & LGMD2E ( $\beta$-Sarkoglykanopathie) & $\beta$-Sarkoglykan \\
\hline & LGMD2F (ס-Sarkoglykanopathie) & $\delta$-Sarkoglykan \\
\hline & LGMD2G (Telethoninopathie) & Telethonin \\
\hline & LGMD2H & TRIM32 \\
\hline & & (E3-Ubiquitin-Ligase) \\
\hline & LGMD2I & FKRP \\
\hline & & (Fukutin-assoziiertes \\
\hline & & Protein) \\
\hline & LGMD2] (Titinopathie) & Titin \\
\hline \multirow[t]{6}{*}{ Distale Myopathien } & Welander-Myopathie & $?$ \\
\hline & Markesberry-Griggs-Myopathie & $?$ \\
\hline & Udd-Myopathie (Titinopathie) & Titin \\
\hline & Laing-Myopathie & $?$ \\
\hline & Nonaka-Myopathie (HIBM2) & GNE \\
\hline & $\begin{array}{l}\text { Miyoshi-Myopathie } \\
\text { (Dysferlinopathie) }\end{array}$ & Dysferlin \\
\hline Kongenitale & CMD mit primärem & Laminin $\alpha 2$ (Merosin) \\
\hline \multirow[t]{12}{*}{ Muskeldystrophien (CMD) } & $\begin{array}{l}\text { Merosin-Mangel } \\
\text { (Merosinopathie / MDC1A) }\end{array}$ & \\
\hline & CMD mit sekundärem & $?$ \\
\hline & Merosin-Mangel 1 (MDC1B) & \\
\hline & CMD mit sekundärem & FKRP \\
\hline & Merosin-Mangel 2 (MDC1C) & \\
\hline & $\begin{array}{l}\text { CMD mit Integrin } \alpha 7 \text {-Mangel } \\
\text { (Integrinopathie) }\end{array}$ & Integrin $\alpha 7$ \\
\hline & Fukuyama-CMD (Fukutinopathie) & Fukutin \\
\hline & Muscle-eye-brain Erkrankungen & \\
\hline & (Santavouri-CMD, & POMT1 \\
\hline & Walker-Warburg-Syndrom) & (O-Mannosyltransferase 1) \\
\hline & $\begin{array}{l}\text { Ullrich-CMD (UCMD / Collagen- } \\
\text { opathie / sklero-atonische } \\
\text { Muskeldystrophie) }\end{array}$ & Collagen Vla2 \\
\hline & CMD mit rigid spine (RSMD1) & SEPN1 (Selenoprotein 1) \\
\hline$?$ & Fazioskapulohumerale MD (FSHD) & $?$ \\
\hline$?$ & Okulopharyngeale MD (OPMD) & Poly-A-bindendes Protein 2 \\
\hline$?$ & $\begin{array}{l}\text { Bethlem-Myopathie } \\
\text { (Collagenopathie) }\end{array}$ & Collagen Vla1-3 \\
\hline
\end{tabular}


Schwerpunkt

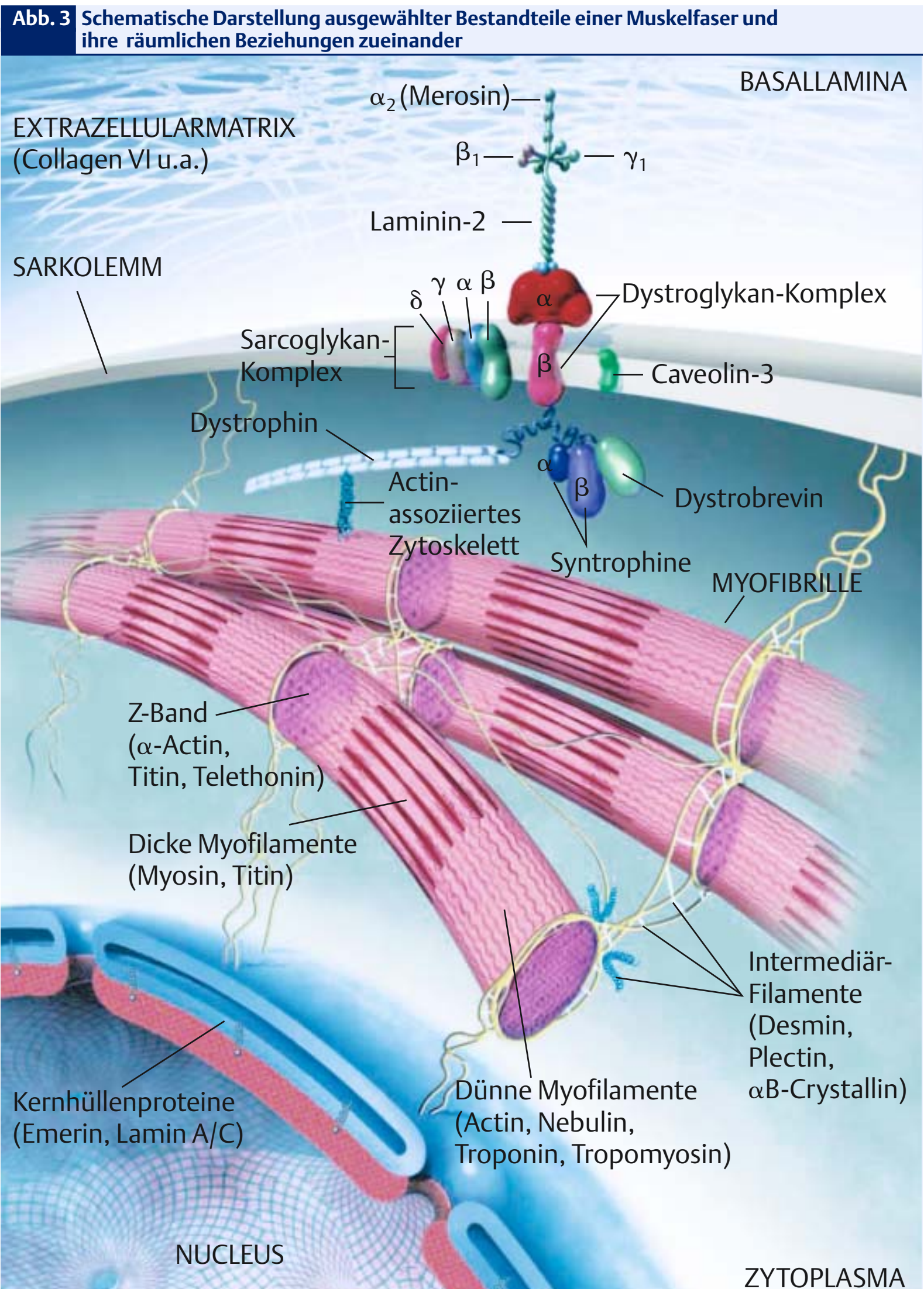

Modif. nach Dalakas MC et al., New England Journal of Medicine 2000; 342: 770-780, mit freundlicher Genehmigung des Verlags 
zeichnet (z.B. MELAS- Syndrom). Das zunehmende Verständnis hormoneller Veränderungen sowie die immer exakteren Möglichkeiten der klinisch-chemischen Labordiagnostik zeigten, dass die Skelettmuskulatur über die bekannten Schilddrüsenerkrankungen hinaus auch durch andere endokrine Veränderungen geschädigt werden kann.
Die generelle Erweiterung des pharmakologisch-therapeutischen Spektrums in der Medizin führte zu der Erkenntnis, dass viele Medikamente neben ihrer erwünschten spezifischen Wirkung mit unterschiedlichen Pathomechanismen zu einer Vielzahl medikamentöstoxischer Myopathien führen können.
Die Identifikation des Strukturproteins Dystrophin als Ursache einer Muskeldystrophie im Jahre 1987 (2) stellte einen Meilenstein in der Myologie und zugleich einen Wendepunkt in der bis dahin üblichen Nomenklatur und Klassifikation von Myopathien dar. Mit dieser bahnbrechenden Entdeckung war es erstmals möglich, einer Muskelkrankheit das

\section{Tab. 2 Hereditäre Myopathien -Teil II: Myopathien mit Strukturbesonderheiten (kongenitale Myopathien)}

\begin{tabular}{|ll}
\hline Krankheit & Gendefekt \\
\hline Nemaline-Myopathien & NEM1 = TPM3 ( $\alpha$-Tropomyosin) \\
& NEM2 = Nebulin \\
& NEM3 = ACTA1 ( $\alpha$-Actin) \\
& NEM4 = TPM2 ( $\beta$-Tropomyosin) \\
& NEM5 = TNNT1 (Troponin T) \\
\hline Central core-Myopathie & RyR1 (Ryanodinrezeptor 1$)$ \\
\hline Multicore/Minicore-Myopathie & RyR1 \\
& SEPN1 \\
\hline zentronukleäre Myopathie & MYF6 (Myogener Faktor 6$)$ \\
\hline myotubuläre Myopathie & MTM1 (Myotubularin) \\
\hline kongenitale Fasertypendisproportion & $?$ \\
\hline myofibrilläre Myopathien & Desmin \\
(Desmin-Myopathien) & $\alpha B-C r y s t a l l i n$ \\
\hline Myopathie mit tubulären Aggregaten & $?$ \\
\hline
\end{tabular}

\section{Tab. 3 Hereditäre Myopathien -Teil III: Myotone Myopathien und lonenkanalkrankheiten}

\begin{tabular}{|c|c|c|}
\hline Untergruppe & Krankheit & Gendefekt \\
\hline \multirow[t]{4}{*}{ myotone Myopathien } & myotone Dystrophie (DM1) & DMPK \\
\hline & & (Myotonin-Protein-Kinase) \\
\hline & proximale myotone Myopathie & ZNF9 (Zink-Finger-Protein 9) \\
\hline & (PROMM/DM2) & \\
\hline \multirow[t]{3}{*}{ Chloridkanal-Myotonien } & Myotonia congenita Thomsen & CLC1 (muskulärer \\
\hline & & Chloridkanal) \\
\hline & Myotonia congenita Becker & CLC1 \\
\hline \multirow[t]{6}{*}{ Natriumkanal-Myotonien } & hyperkaliämische periodische Paralyse & SCN4A \\
\hline & & ( $\alpha$-Untereinheit des \\
\hline & & Natriumkanals) \\
\hline & hypokaliämische periodische Paralyse 2 & SCN4A \\
\hline & Paramyotonia congenita Eulenburg & SCN4A \\
\hline & Natrium-sensitive Myotonie & SCN4A \\
\hline \multirow[t]{2}{*}{ Kalziumkanal-Myotonien } & hypokaliämische periodische Paralyse 1 & DHPR (Dihydropyridin- \\
\hline & & Rezeptor) \\
\hline \multirow[t]{4}{*}{ Kaliumkanal-Myotonien } & Andersen-Syndrom (periodische Paralyse & $\mathrm{KCJN2}$ \\
\hline & mit Herzrhythmusstörungen) & (Kaliumkanal-assoziiertes \\
\hline & & Peptid) \\
\hline & hypokaliämische periodische Paralyse 3 & $\mathrm{KCJN2}$ \\
\hline \multirow[t]{6}{*}{$?$} & maligne Hyperthermie & MHS1 = RyR1 \\
\hline & & MHS2 (SCN4A?) \\
\hline & & MHS3 \\
\hline & & MHS4 \\
\hline & & MHS5 = DHPR \\
\hline & & MHS6 \\
\hline
\end{tabular}


mutierte Genprodukt zuzuordnen. In den folgenden anderthalb Jahrzehnten gelang dies für eine große Zahl hereditärer Myopathien, wobei unterschiedliche zelluläre Bestandteile (z.B. Sarkolemm, Kernmembran, Myofibrillen, Intermediärfilamente, Extrazellulärmatrix) betroffen sein können (Abb. 3). Bei vielen anderen Muskelerkrankungen ist bisher lediglich der Genort bekannt.

Die Konsequenzen für die Bezeichnung von hereditären Muskelkrankheiten sind erheblich. Aktuell befin- den wir uns in einer noch nicht abgeschlossenen Phase, in der man vielfach dazu über geht, Myopathien nach dem jeweils zugrunde liegenden Protein- bzw. Gendefekt zu benennen, wobei aktuell die herkömmliche und die „moderne“ Bezeichnung häufig noch synonym verwendet werden (6). Da einerseits phänotypisch sehr ähnliche Myopathien auf völlig verschiedenen Proteindefekten beruhen können und andererseits verschiedene Mutationen im selben Gen und sogar ein und dieselbe Mutation zu völlig unterschiedlichen klinischen Syndromen führen können, wird dies auch Auswirkungen auf die Klassifikation von Myopathien haben. Diesbezüglich ist anzunehmen, dass die bisherige Praxis des Zusammenfassens von Muskelerkrankungen nach klinischen, pathologischen, elektromyographischen bzw. biochemischen Kriterien (4) ebenfalls einer an der Lokalisation bzw. Funktion der jeweils mutierten Proteine orientierten Ordnung weichen wird (5).

\section{Tab. 4 Hereditäre Myopathien -Teil IV: Metabolische Myopathien}

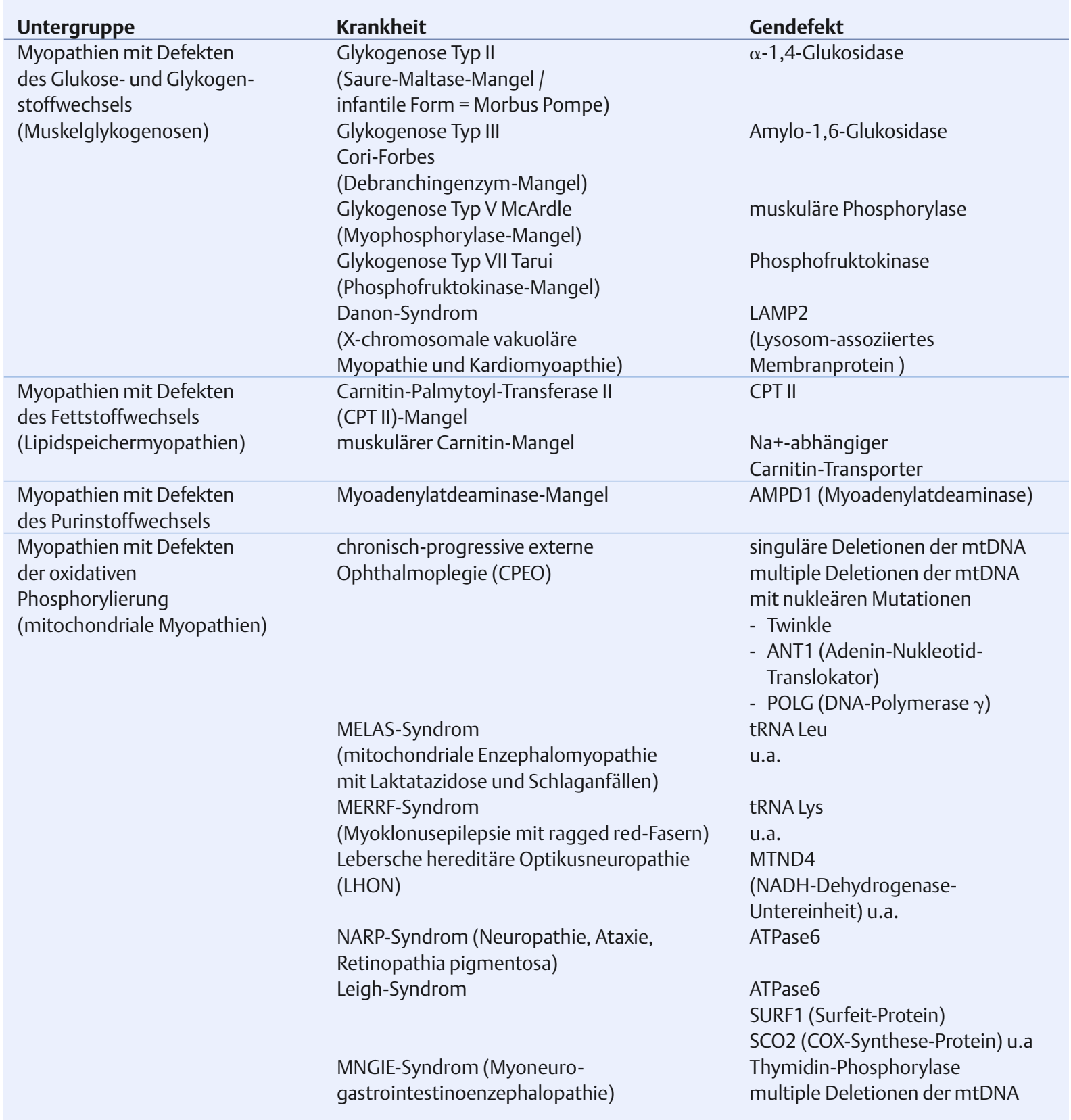


Die keinen Anspruch auf Vollständigkeit erhebenden Tabellen 1-6 stellen den Versuch eines Kompromisses dar, Elemente einer traditionellen Unterteilung/Klassifikation mit - soweit möglich - an den pathophysiologischen Grundlagen orientierten Krankheitsbezeichnungen zu verbinden.

Die große Vielfalt von ganz unterschiedlichen Myopathien macht deutlich, dass eine moderne Diagnostik von Patienten mit offensichtlichen Muskelerkrankungen nicht nur auf die Elektrophysiologie, CKBestimmung und herkömmliche histologische Untersuchung beschränkt sein kann, sondern ein umfassendes Repertoire an immunhistochemischen, biochemischen und molekularbiologischen Methoden erfordert $(7,9)$.

\section{Summary}

In the past, definition and classification of myopathies have been categorized by their clinical appearance, by their original describer, by age, by histological hallmarks or by a mix of these criterias. Due to the progress in understanding the molecular basis of

\section{Tab. 5 Hereditäre Myopathien unklarer Zuordnung}

\begin{tabular}{ll} 
Krankheit & Gendefekt \\
\hline Brody-Syndrom & SERCA1 \\
& (sarkoplasmatische \\
& Ca2+-ATPase) \\
Barth-Myopathie & Taffazzin \\
McLeod-Syndrom & XK (Membrantransporter) \\
Rippling-Syndrom & Caveolin 3 \\
Schwartz-Jampel-Syndrom & Perlecan
\end{tabular}

\section{Tab. 6 Erworbene Myopathien}

\begin{tabular}{|c|c|}
\hline Krankheit & Krankheit bzw. Noxe \\
\hline \multirow[t]{4}{*}{ Entzündliche Muskelkrankheiten } & Polymyositis \\
\hline & Dermatomyositis \\
\hline & Einschlusskörpermyositis \\
\hline & Erreger-bedingte Myositiden \\
\hline \multirow[t]{10}{*}{ Endokrine Myopathien } & hyperthyreote Myopathie \\
\hline & (endokrine Ophthalmoplegie) \\
\hline & hypothyreote Myopathie \\
\hline & (Hoffmann-Syndrom) \\
\hline & hyperparathyreote Myopathie \\
\hline & hypoparathyreote Myopathie \\
\hline & Nebennierenrinden-induzierte \\
\hline & Myopathie \\
\hline & Akromegalie-induzierte \\
\hline & Myopathie \\
\hline \multirow[t]{9}{*}{ Medikamentös-toxische Muskelschädigung } & Alkohol \\
\hline & AZT \\
\hline & Chloroquin \\
\hline & Cyclosporin \\
\hline & Herbizide \\
\hline & Kortikosteroide \\
\hline & Lipidsenker \\
\hline & Neuroleptika \\
\hline & $\begin{array}{l}\text { Organophosphatester } \\
\text { uvm. }\end{array}$ \\
\hline \multirow[t]{2}{*}{ Paraneoplastische Muskelschädigung } & Amyloid-Myopathie \\
\hline & Decorin-Myopathie \\
\hline
\end{tabular}

myopathies it is now possible to link most of the hereditary disorders of skeletal muscle to their pathogenetic mutations. As a consequence, the majoritiy of muscle diseases now can be nominated and classified based on their genetic abnormalities or protein defects.

\section{Key words}

Myopathy - neuromuscular disorders - classification

\section{Literatur}

1. Erb W: Dystrophia muscularis progressiva. Klinische und pathologisch-anatomische Studien. Dt Z Nervenheilkunde 1891; I: 13-94, 173-261

2. Hoffman EP, Brown RH Jr, Kunkel LM: Dystrophin: the protein product of the Duchenne muscular dystrophy locus. Cell 1987; 51: 919-928

3. Holt IJ, Harding AE, Morgan-Hughes JA: Deletions of muscle mitochondrial DNA in patients with mitochondrial myopathies. Nature 1988; 311: 717-719

4. Karpati G, Hilton-Jones D, Griggs RC (eds): Disorders of voluntary muscle. Cambridge, Cambridge University Press, 7th ed. 2001, 374-84

5. Karpati G (ed.): Structural and molecular basis of skeletal muscle diseases. Basel, ISN Neuropath Press, 2002

6. Neuromuscular Disorders: Gene Location Table: www.elsevier.com/locate/nmdgenetable

7. Pongratz D, Zierz S (Hrsg.): Neuromuskuläre Erkrankungen. Köln, Deutscher Ärzteverlag, 2003 (im Druck)

8. Walton JN, Natrass FJ: On the classification, natural history and treatment of the myopathies. Brain 1954; 77: 169-230

9. Zierz S, Jerusalem F: Muskelkrankheiten. Stuttgart, Georg Thieme Verlag, 3. Aufl. 2003 (im Druck)

\section{Korrespondenzadresse:}

Dr. S. Neudecker

Klinik und Poliklinik für Neurologie

Martin-Luther-Universität Halle-Wittenberg

Ernst-Grube-Str. 40

D-06097 Halle/Saale

e-mail:

stephan.neudecker@medizin.uni-halle.de 